\title{
Evaluation of sorption capabilities of biopolymeric microspheres by the solid-phase extraction
}

\author{
M. Goliszek $k^{1}$ M. Sobiesiak ${ }^{1} \cdot$ K. Fila ${ }^{1} \cdot$ B. Podkościelna ${ }^{1}$
}

Received: 25 October 2018 / Revised: 7 December 2018 / Accepted: 3 January 2019 / Published online: 10 January 2019

(c) The Author(s) 2019

\begin{abstract}
This study presents the sorption capabilities of biopolymeric microspheres with commercial kraft lignin. The suspensionemulsion polymerization method was employed to obtain microspheres in spherical forms. Before copolymerization lignin was modified with methacryloyl chloride to increase its affinity for monomers. Two different types of microspheres (hybrid and non-hybrid) with the increasing quantity of lignin were studied. In the hybrid ones, divinylbenzene (DVB) and methacrylated kraft lignin (L) were used as organic compounds whereas triethoxyvinylsilane (TEVS) was applied as an inorganic one. The second type of microspheres was synthesized without the addition of TEVS. There were also applied two materials without a lignin component for comparison purposes. The hybrid lignin-containing microspheres exhibited a more developed porous structure than the non-hybrid ones. Their specific surface areas were in the range of $454-522 \mathrm{~m}^{2} / \mathrm{g}$ and $2305 \mathrm{~m}^{2} / \mathrm{g}$, respectively. The results of swelling studies of synthesized materials in typical organic solvents and distilled water were presented. Additionally, in acetone and water, tendency to swell depending on time has been determined. The prepared microspheres were tested as sorbents for removal of fluorine containing phenolic compounds from the aqueous solutions using the solid phase extraction (SPE) technique. A mixture of: phenol ( $\mathrm{Ph}$ ), 2-fluorophenol (2-FPh), 2,6-difluorophenol $(2,6-\mathrm{DFPh})$ and 2,4,6-trifluorphenol (2,4,6-TFPh) as testing compounds was applied. The prepared porous polymers possessed good sorption properties towards the testing compounds.
\end{abstract}

Keywords Kraft lignin $\cdot$ Triethoxyvinylsilane $\cdot$ Polymerization $\cdot$ Hybrid microspheres $\cdot$ Porous structure $\cdot$ SPE

\section{Introduction}

Nowadays an increasing number of toxic organic compounds which are discharged into the wastewaters is observed. Among all the pollutants, pesticides, dyes and phenolics are of particular concerned because of their persistency in the environment and/or extreme toxicity (Aksu 2005; Yu et al. 2014; Torres et al. 2003; Sostar-Turk et al. 2005).

Phenolic compounds are hazardous wastes released into the aquatic environment by petroleum refineries, petrochemical industries, phenolic resins, coke ovens in the steel plants as well as chemical, pharmaceutical, dye industries and fertilizers. The quantity of the phenolic compounds in the industrial wastewaters is often much larger than the established

M. Goliszek

marta.goliszek@poczta.umcs.lublin.pl

1 Department of Polymer Chemistry, Faculty of Chemistry, Maria Curie-Skłodowska University, M. Curie-Skłodowskiej Sq. 5, 20-031 Lublin, Poland standard limits (Ahmaruzzaman and Sharma 2005). Human consumption of water contaminated with phenols can damage capillaries which may result in organism death (Aksu 2005; Montaño 2013).

The analytical techniques commonly used for determination of phenols are capillary electrophoresis (CE) and highperformance liquid chromatography (HPLC) combined with fluorescence and ultraviolet detection, mass spectroscopy or electrochemical detection. Also gas chromatography (GC) with electron-capture detection, flame ionization detection, mass spectroscopy or microwave-induced plasma atomic emission spectroscopy are common tools in the phenols analysis. Unfortunately, none of these combinations could achieve quantification limits, required for direct determination of phenols in the drinking water. This makes that preconcentration is a necessary step in the analytical process. For determination of phenols in water the liquid-liquid extraction (LLE) is used as a preconcentration step but the tendency is to replace it by the solid-phase extraction (SPE) and solid-phase microextraction (SPME). Various reasons 
for this innovation are, inter alia, foam formation, a large volume of organic solvents, problems with automation of LLE processes and length of analysis time. SPE is easy to automate, requires smaller portions of organic solvents and gives a possibility of integration into on-line dedicated systems (Rodriguez et al. 2000; Podkościelna and Sobiesiak 2016; Sobiesiak et all. 2006; Puziy et al. 2010).

Retention of polar phenols on the polymeric sorbents in the SPE method can be improved by introduction of polar groups into the polymer. Due to the hydrophilic character of functional groups there is improvement in the wetting characteristics. It supports mass transfer of polar phenols to the sorbent from the water solution (Rodriguez et al. 2000).

The recent methods applied for phenolics removal from wastewaters are: adsorption on activated carbons, microbial degradation, chemical oxidation, deep-well injection, solvent extraction, incineration and irradiation. Lately the most extensive research has been carried out using activated carbons but their use as the adsorbents for the industrial wastewater treatment is very capital-intensive. There is also a problem with regeneration of activated carbon, high cost of manufacture and intraparticle resistance in the processes of adsorption in practice. Therefore the alternative technologies have to be explored (Aksu 2005; Ahmaruzzaman and Sharma 2005; Carrott et al. 2005; Stoeckli et al. 2007; Mourao et al. 2006; Dąbrowski et al. 2005).

A promising alternative to supplement or replace the present processes of removing organic pollutants from wastewaters is biosorption (Aksu 2005). This is the process of organic and inorganic compounds, metal or metalloid species and particulates removal from the solution using biological material (Rajput and Yanning 2015). The main advantages of biosorption are: efficiency, high selectivity, good removal performance and also cost effectiveness. The raw materials which are either wastes from industrial processes or abundant in nature can be used as biosorbents having performances whose action is often comparable with those of the ion exchange resins (Aksu 2005; Braghiroli et al. 2018; Wu and Yu 2006; Navarro et al. 2009; Kumar et al. 2018; Veglio and Beolchini 1997; Demirbas 2008; Sciban et al. 2011; Suhas et al. 2007). The use of adsorbents, which are a part of natural environment, eliminates generation of additional pollution.

The most commonly used biosorbents are: shells of invertebrates, peat, straw, fern, zeolites and lignin (Wysokowski et al. 2014). Among them lignin is one of the most abundant natural aromatic polymers on the Earth, which is synthesized by all land plants. This is a constituent of lignocellulosic biomass. Chemically, it is a complex phenolic biopolymer, which contains aromatic alcohols and a lot of polar functionalities. Lignin is a three-dimensional phenylpropanoid polymer linked by ether bonds between the monomeric phenylpropane units.
A huge amount of lignin is derived as a waste product from the wood processing in the paper and pulp industry. It is burned to generate mainly energy.

However, lignin is characterized by some properties such as: non-toxic nature, very versatile performance, high value potential, being available and inexpensive in huge amounts which makes it increasingly important in numerous biotechnological applications, such as a tanning agent, adhesive, adsorbent of dyes, metal ions, surfactants, organic pollutants like pesticides and phenols. Lignin possesses a lot of very reactive centers which can be modified in chemical, physical and enzymatic reactions, being a huge potential for their exploitation as the industrial raw materials. What is more, lignin contains numerous oxygen (alcoholic and phenolic hydroxyl, ether, methoxyl) functionalities might be responsible for the sorptive capacity. Lignin as one of the lignocellulosic materials can be used as an effective and resistant sorbent for the removal of many different potential pollutants from wastewaters. It is characterized by mechanical resistance, hydrophilic character, easy functionalization and the possibility to be used in different regimes and forms (Peterson 2012; Ciesielczyk et al. 2017; Bartczak et al. 2017; Atifi et al. 2017; Klapiszewski et al. 2014a, b; Cuia et al. 2015; Xu et al. 2015; Severtson and Banerjee 1996; Xiny et al. 1994; Mamleeva and Lunin 2016; Abdolaki et al. 2014; Demirbas 2004; Gue et al. 2008; Dizhbit et al. 1999; Da Silva et al. 2011; Albadarin et al. 2011).

Raw lignin is a nonporous material but its addition as a component to the porous polymers allows to create a material which due to the well-developed porous structure and the presence of functional groups can find applications in many sorption processes (Podkościelna et al. 2015, 2017a, 2017b; Goliszek et al. 2018).

In the previous paper the microspheres with styrene, DVB and kraft lignin were synthesized. Lignin was modified with acrylic acid and epichlorohydrin along with acrylic acid. These materials had a limited sorption efficiency. The highest recovery values in the SPE experiments reached only 47\% (Podkościelna et al. 2015). The reason for this low efficacy was partial blocking of the polymer porous structure by huge lignin particles, thus in that case lignin acted more as a filler.

The aim of the current research was the application of porous materials composed of modified kraft lignin, DVB and DVB with TEVS as a sorbents for removal of phenolic pollutants from the aqueous solutions using the solid phase extraction (SPE) technique.

Additionally, different amounts of lignin were added to the polymeric systems to improve hydrophilic character of the obtained materials. The comparison of chemical structures, thermal properties, swelling, morphology of two kinds of materials (with/without TEVS) as well as and the sorption of these materials are analyzed here in detail. 


\section{Experimental}

\subsection{Chemicals and eluents}

Triethoxyvinylsilane (TEVS), bis(2-ethylhexyl)sulfosuccinate sodium salt and benzyl alcohol were from Sigma-Aldrich. $\alpha$ '-Azoiso-bis-butyronitrile (AIBN) and divinylbenzene (DVB) (62.2\% of 1,4-divinylbenzene, $0.2 \%$ of 1,2-divinylbenzene, and ethylvinylbenzene were washed with $3 \%$ aqueous sodium hydroxide solution before the use) were obtained from Merck (Darmstadt, Germany). Acetone, methanol, chloroform, toluene, tetrahydrofuran (THF), toluene, decan-1-ol and acetonitrile were obtained from Avantor Performance Materials (Poland).

In our study commercial available kraft lignin from Sigma-Aldrich was applied. Before the copolymerization, lignin was modified with methacryloyl chloride as described by Podkościelna et al. 2017b.

\subsection{Synthesis of microspheres}

For the SPE experiment the microspheres based on DVB (as a crosslinking monomer) were selected. Two groups of materials with modified lignin were tested. First ones, consisted of DVB and methacrylated kraft lignin (L) and second ones, made of DVB, TEVS and L.

Now, the briefly description on synthesis is presented, the detailed information are presented in Ref. (Podkościelna et al. 2017b; Goliszek et al. 2018).

The synthesis of polymeric microspheres was performed in the aqueous medium. Redistilled water and bis(2-ethylhexyl)sulfosuccinate sodium salt (surfactant) were stirred for $0.5 \mathrm{~h}$ at $80{ }^{\circ} \mathrm{C}$ in a $250 \mathrm{~cm}^{3}$ three-necked flask fitted with a stirrer, a water condenser and a thermometer. Then, the solutions containing DVB, (or DVB and TEVS), different amounts of lignin (Table 1), the initiator AIBN (1 wt \%) and the pore-forming diluents were added while stirring. Due

to the poor solubility of lignin, pore-forming diluents were also solvents for lignin. The reaction mixture was stirred at $350 \mathrm{rpm}$ for $18 \mathrm{~h}$ at $80^{\circ} \mathrm{C}$. The obtained microspheres were washed with distilled hot water ( 2 L), filtered off, dried and extracted in a Soxhlet apparatus with boiling acetone. After drying, the microspheres were fractionated with sieves.

\subsection{Polymers characterization}

Attenuated total reflectance (ATR) FTIR spectra were recorded using a Bruker TENSOR 27 FTIR spectrophotometer (resolution of $4 \mathrm{~cm}^{-1}, 32$ scans accumulated).

Differential scanning calorimetry (DSC) measurements were performed with a Netzsch DSC 204 calorimeter (Germany) operated in the dynamic mode. DSC measurements were made using aluminum pans with pierced lids and a sample mass of $\sim 5-10 \mathrm{mg}$ under a nitrogen atmosphere $\left(30 \mathrm{ml} \mathrm{min}{ }^{-1}\right)$. Dynamic scans were performed at a heating rate of $10{ }^{\circ} \mathrm{C} \min ^{-1}$ in the temperature range of $20-500{ }^{\circ} \mathrm{C}$. The parameters such as: the range of decomposition temperatures $\left(\mathrm{T}_{\text {onset, }} \mathrm{T}_{\text {offset }}\right)$ and maximum decomposition temperature $\left(T_{d}\right)$ were determined.

The appearances and morphologies of the microspheres were studied using MORPHOLOGI G3 Malvern optical microscope (Great Britain).

The porous structures of the copolymers were characterized by $\mathrm{N}_{2}$ adsorption at $-196{ }^{\circ} \mathrm{C}$ (ASAP 2405 adsorption analyzer, Micrometrics Inc., USA). Prior to the analysis, the copolymers were degassed at $140{ }^{\circ} \mathrm{C}$ for $2 \mathrm{~h}$. The specific surface area was calculated according to the BrunauerEmmett-Teller (BET) method with the assumption that the area occupied by a single nitrogen molecule is $16.2 \AA^{2}$. The pore volumes and pore size distributions were determined by the Barrett-Joyner-Halenda (BJH) method.

The swelling coefficients (B) were determined using equilibrium swelling in acetone, acetonitrile, methanol, toluene, chloroform, THF, and distilled water. B is expressed as:

$$
B=\frac{V_{s}-V_{d}}{V_{d}} \cdot 100 \%
$$

Table 1 Experimental parameters of the syntheses

\begin{tabular}{|c|c|c|c|c|c|c|c|}
\hline \multirow[t]{2}{*}{ Polymer } & \multicolumn{4}{|c|}{ Monomers (g) } & \multicolumn{3}{|c|}{ Diluents $\left(\mathrm{cm}^{3}\right)$} \\
\hline & DVB [g] & TEVS [g] & $\mathrm{w} / \mathrm{w} \% \mathrm{~L}$ & w/w $\%$ on $\mathrm{CM}$ & BA & $\mathrm{T}$ & $\mathrm{D}$ \\
\hline $1 \mathrm{LNH}$ & 5 & 0 & 17 & 17 & 14 & - & - \\
\hline $2 \mathrm{LNH}$ & 5 & 0 & 33 & 33 & 14 & - & - \\
\hline $3 \mathrm{LNH}$ & 5 & 0 & 50 & 50 & 14 & - & - \\
\hline OLNH & 5 & 0 & 0 & 0 & 10 & - & - \\
\hline $1 \mathrm{LH}$ & 5 & 3.6 & 12 & 20 & - & 5 & 5 \\
\hline $2 \mathrm{LH}$ & 5 & 3.6 & 17 & 30 & - & 5 & 5 \\
\hline $3 \mathrm{LH}$ & 5 & 3.6 & 23 & 40 & - & 5 & 5 \\
\hline $\mathrm{OLH}$ & 5 & 3.6 & 0 & 0 & - & 5 & 5 \\
\hline
\end{tabular}

$L$ lignin, $L H$ lignin hybrid materials, $L N H$ lignin non hybrid materials, $B A$ benzyl alcohol, $T$ toluene, $D$ decan-1-ol, $C M$ crosslinking monomer 
where $V_{s}$ is the volume of a sample after swelling and $V_{d}$ is the volume of a dry sample.

\subsection{Solid phase extraction experiments}

The off-line SPE method was used for the estimation of sorption capabilities of the obtained microspheres towards phenol and its fluorinated derivatives. The mixture of phenols was preconcentrated from the aqueous solution using laboratory cartridges filled with $100 \mathrm{mg}$ of the studied material. The multi-component aqueous solutions of phenols were prepared by dilution $(1: 50 \mathrm{v} / \mathrm{v})$ of the standard methanolic solution containing $100 \mathrm{mg} / \mathrm{L}$ of: phenol $(\mathrm{Ph})$, 2-fluorophenol (2-FPh), 2,6-difluorophenol (2,6-DFPh), and 2,4,6-trifluorophenol (2,4,6-TFPh). The increasing sample volumes of the aqueous solutions were sucked through the cartridge using the water aspirator. Then the phenolic compounds were eluted with methanol $(2 \mathrm{~mL}$ of methanol for each $100 \mathrm{~mL}$ of sucked sample volume). Concentration of phenols in the obtained eluates was determined with the HPLC method. The details of the analysis were described in the previous papers (Sobiesiak et al. 2006; Puziy et al. 2010; Podkościelna and Sobiesiak 2016). Recovery values were calculated as the mean of three measurements. Decrease of the recovery below $25 \%$ of the maximum value was assumed as a breakthrough volume for the compound under consideration.

\section{Results and discussion}

In Fig. 1 the chemical structures of the monomers used in the suspension-emulsion copolymerization are shown. The experimental parameters of two different systems with the increasing lignin (L) content are described in detail in Table 1. Copolymers without TEVS are described as lignin non hybrid materials (LNH) and with TEVS as lignin hybrid materials (LH). Weight percentage (w/w \%) of lignin content was in the range $12-50 \mathrm{w} / \mathrm{w} \%$ depending on the monomers used in the systems (mono- DVB or dual- DVB-TEVS).
Figure 2 presents the ATR/FT-IR spectra of the copolymers with and without TEVS. For all of them bands corresponding to: the aromatic $\mathrm{C}=\mathrm{C}$ bonds (at $1509-1510 \mathrm{~cm}^{-1}$ ), the aromatic system (at 1450, 1490, 1650-1600 $\mathrm{cm}^{-1}$ ), $\mathrm{C}-\mathrm{H}$ vibrations in the armatic rings $\left(900-650 \mathrm{~cm}^{-1}\right)$ and vinyl groups (at $900-830 \mathrm{~cm}^{-1}$ ) are visible. For the lignincontaining materials a band at $1728-1732 \mathrm{~cm}^{-1}$ produced by carbonyl groups is observed. What is more, a broad band at $3243 \mathrm{~cm}^{-1}$ from the hydroxyl groups is not visible for the samples with lignin. These observations demonstrate the successful modification of lignin with methacrylate groups. In the spectra of the microspheres with TEVS a strong band at $1000-1110 \mathrm{~cm}^{-1}$ corresponding to the vibrations of $\mathrm{Si}-\mathrm{O}-\mathrm{C}$ groups is observed which confirms incorporation of TEVS into the synthesized polymeric materials. It is worth mentioning that the band characteristic of the water content $\left(3500-3200 \mathrm{~cm}^{-1}\right)$ in all the studied microspheres is small even for the samples containing the largest amount of lignin.

The thermal properties of the copolymers were investigated by the DSC method. The DSC curves and data are presented in Fig. 3a, b and Table 2, respectively. On the curves there is a small exothermic effect at about $170{ }^{\circ} \mathrm{C}$ (Fig. 3a) associated with the process of crosslinking. This effect is typical for DVB derivatives. In Fig. $3 b$ one visible endothermic effect $\left(429-449^{\circ} \mathrm{C}\right)$ associated with the thermal degradation process of the sample is observed. With the increasing content of lignin decomposition takes place at slightly lower temperatures for both systems (with and without TEVS). Analysis of the obtained results leads to the conclusion that the synthesized materials have good thermal resistance.

For chromatographic purposes the sorbent particles should have a uniform spherical shape. This improves the process of sorption because of the mobile phase regular flow that minimalizes the effects of diffusion. In our study the actual shapes of the obtained microspheres were studied by optical microscope (Fig. 4). The copolymers with TEVS have diameters in the range of $20-45 \mu \mathrm{m}$ whereas those without TEVS - in the range of $10-30 \mu \mathrm{m}$. The addition of lignin resulted in significant improvement of spherical shapes and particle sizes of the microspheres

Fig. 1 Scheme of copolymerization

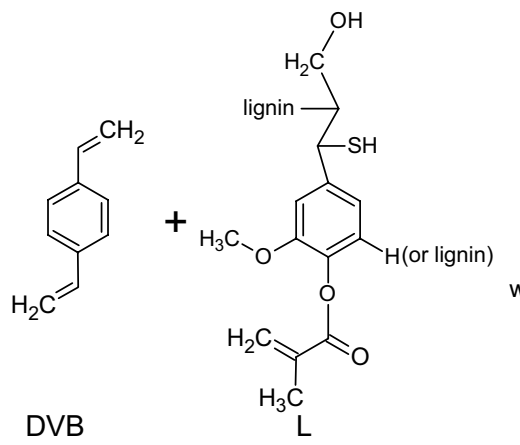

DVB
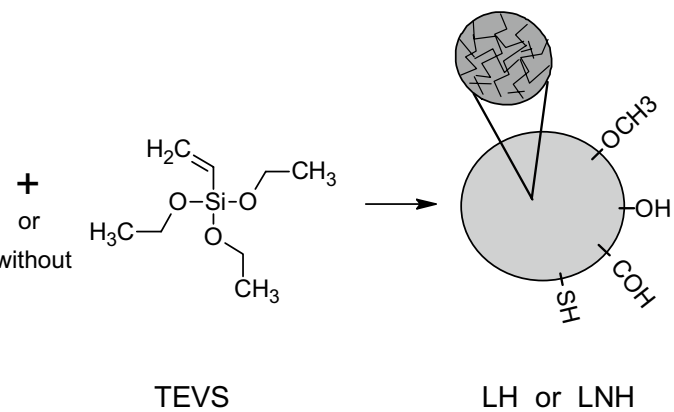

$\mathrm{LH}$ or $\mathrm{LNH}$ 

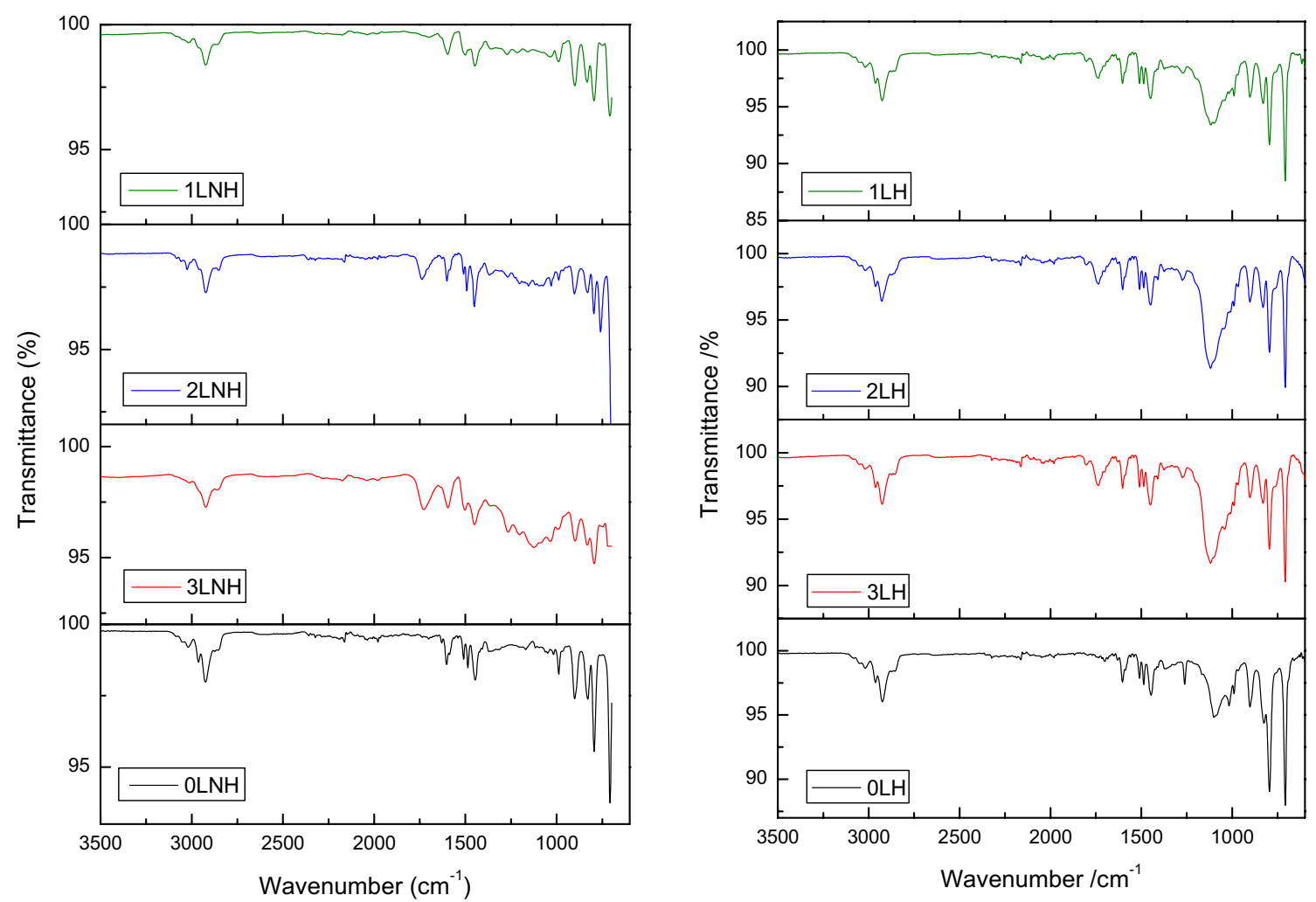

Fig. 2 ATR/FT-IR spectra of the studied materials

in both systems. Among the hybrid materials, the $0 \mathrm{LH}$ copolymer is characterized by the most irregular shapes and the smallest size of the particles. The most homogeneous size distribution and the most uniform spherical shape were obtained for the 1LNH copolymer. A similar situation was observed for the materials without TEVS. All of the copolymers had a tendency to agglomerate.

A well-developed porous structure and the presence of micro- and mesopores are very important factors for the effective sorption processes. For this reason, the investigations of the porosity parameters are necessary. The characteristic parameters of the porous structure of the studied materials are presented in Table 3 . The $S_{B E T}$ and $\mathrm{V}_{\text {TOT }}$ for the copolymers without TEVS are in the range $2-542 \mathrm{~m}^{2} / \mathrm{g}$ and $0.005-1.299 \mathrm{~cm}^{3} / \mathrm{g}$, respectively whereas for the copolymers with TEVS they are in the range $454-522 \mathrm{~m}^{2} / \mathrm{g}$ and $1.040-1.881 \mathrm{~cm}^{3} / \mathrm{g}$. Hybrid materials are characterized by much larger specific surface area in comparison with the non-hybrid ones.

The increase of porosity in hybrid systems is evidently related to the application of TEVS as a monomer. This compound contains in its structure three large ethoxyl groups which occupy a position as far away as possible from the Si atom and thus they limit the formation of a polymer network in their neighborhood.
The decreases in specific surface areas and total pore volumes with the increasing content of lignin are observed for both systems. This is due to partial blocking of the pores by the lignin molecule. All of the studied materials are mesoporous and well suited for the sorption application in the liquid/aqueous systems.

The pore size distribution curves are presented in Fig. 5. For the materials without TEVS the lignin-containing copolymers exhibit narrower pore size distribution in comparison to the OLNH homopolymer. In the series with TEVS the OLH copolymer exhibits the lowest value of average pore diameter. The maximum values of pore size distribution are: $320 \AA, 240 \AA$ and $235 \AA$ for $1 \mathrm{LH}, 2 \mathrm{LH}$ and $3 \mathrm{LH}$, respectively. The greater amount of lignin is added into the material, the narrower size pores distribution is obtained and the pore diameter values are smaller.

Swellability is a factor determining the accessibility of the internal chemical structure in the crosslinked polymer for the penetration by the solvent molecules (Podkościelna and Sobiesiak 2016). The results of the swelling studies for the obtained materials are presented in Table 4. The swellability coefficients (B) were determined using typical organic solvents and distilled water. Among the non-hybrid materials, the smallest tendency to swell is observed for the $0 \mathrm{LNH}$ and $1 \mathrm{LNH}$ polymers. The largest swellability coefficients 

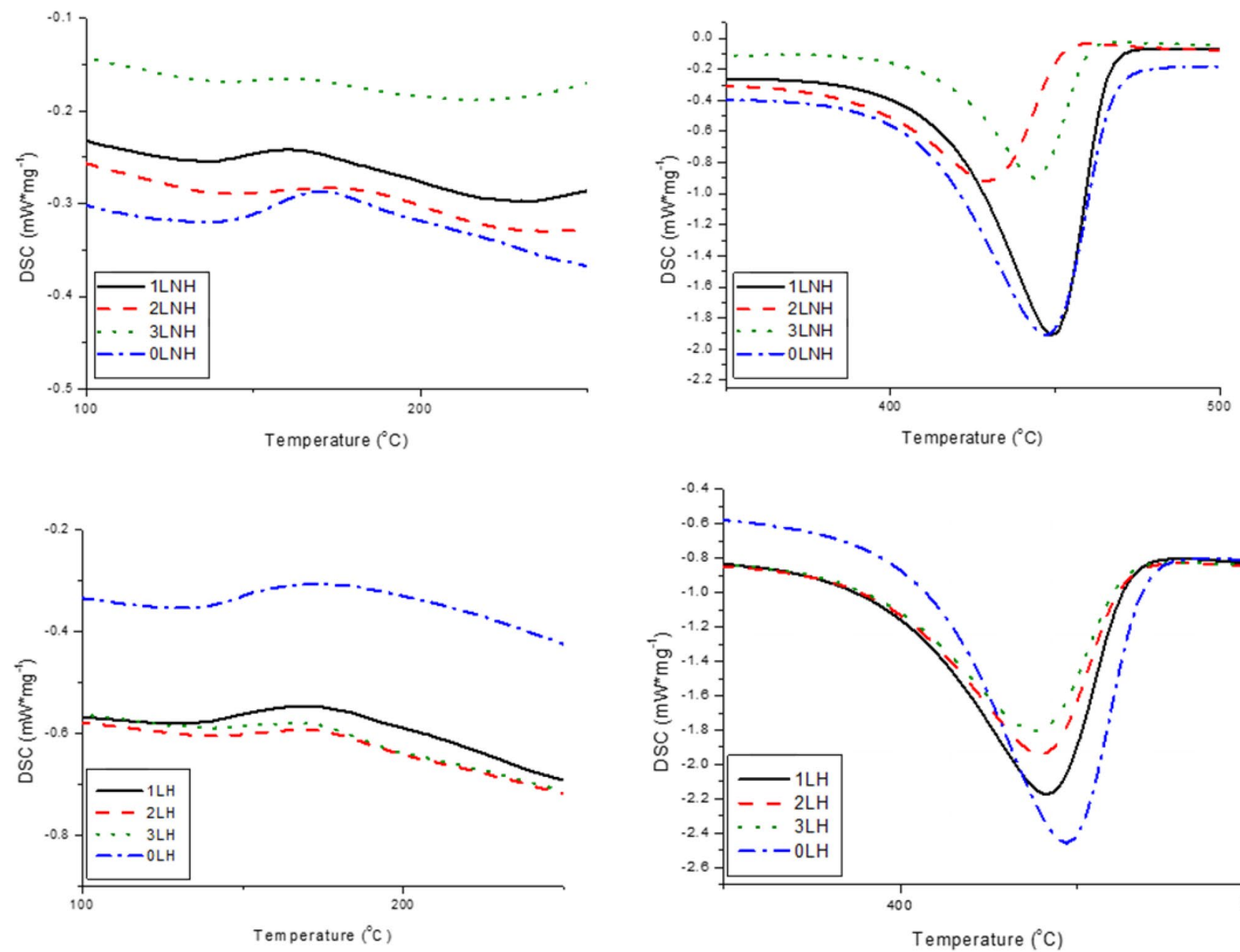

(a)

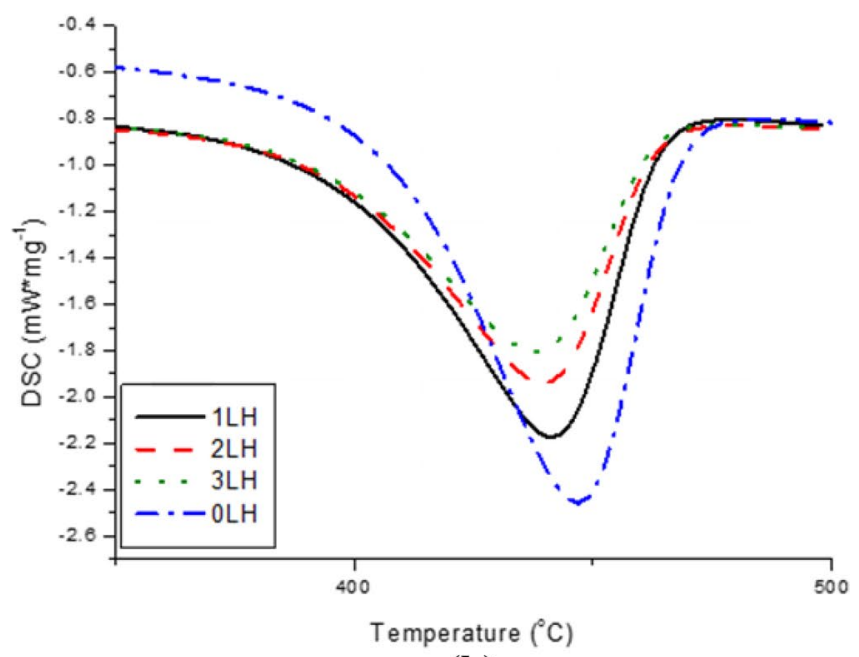

(b)

Fig. 3 DSC curves of the microspheres

Table 2 DSC data for the copolymers

\begin{tabular}{llll}
\hline Polymer & $\mathrm{T}_{\text {onset }}\left({ }^{\circ} \mathrm{C}\right)$ & $\mathrm{T}_{\mathrm{d}}\left({ }^{\circ} \mathrm{C}\right)$ & $\mathrm{T}_{\text {offset }}\left({ }^{\circ} \mathrm{C}\right)$ \\
\hline $1 \mathrm{LNH}$ & 368 & 449 & 478 \\
$2 \mathrm{LNH}$ & 375 & 444 & 468 \\
$3 \mathrm{LNH}$ & 348 & 429 & 461 \\
$0 \mathrm{LNH}$ & 365 & 447 & 481 \\
$1 \mathrm{LH}$ & 343 & 442 & 478 \\
$2 \mathrm{LH}$ & 342 & 440 & 476 \\
$3 \mathrm{LH}$ & 340 & 438 & 474 \\
$0 \mathrm{LH}$ & 355 & 447 & 484 \\
\hline
\end{tabular}

were obtained for the $2 \mathrm{LNH}$ copolymer. All the copolymers without TEVS reached the greatest swellability values in acetone. In distilled water they did not swell at all $(\mathrm{B}=0 \%)$.

As far as the hybrid microspheres are concerned their swellability coefficients values are much lower. The addition of TEVS results in blocking of the swelling process

of the microspheres. This observation can be the result of TEVS non-wettability of typical organic solvents and water. The 0LH copolymer does not have a tendency to swell. The largest swellability coefficients are exhibited by the $2 \mathrm{LH}$ and 3LH copolymers. With the increasing lignin content, the increase in swellability coefficients is evident. The hybrid microspheres reached the greatest tendency to swell in THF and chloroform and the smallest ones in water.

Additionally, in case of water and acetone the swellability coefficients as a function of time were determined and presented in Fig. 6. As one can see non-hybrid microspheres do not swell in aqua dest. at all up to $25 \mathrm{~min}$. After this time the swellability coefficients increase to $2 \%$ and do not change even after $60 \mathrm{~min}$. In acetone, swelling process starts after 1 min and maximum values of swelling are observed after $15 \mathrm{~min}(250 \%$, for $2 \mathrm{LNH}$ and 3LNH). Among hybrid microspheres in aqua dest. swelling starts after $1 \mathrm{~min}$ for $2 \mathrm{LH}$ and $3 \mathrm{LH}$ and it reaches a value of max. $8 \%$. Hybrid material without lignin does not swell up to $15 \mathrm{~min}$. After this time 
Fig. 4 Photographs of the microspheres
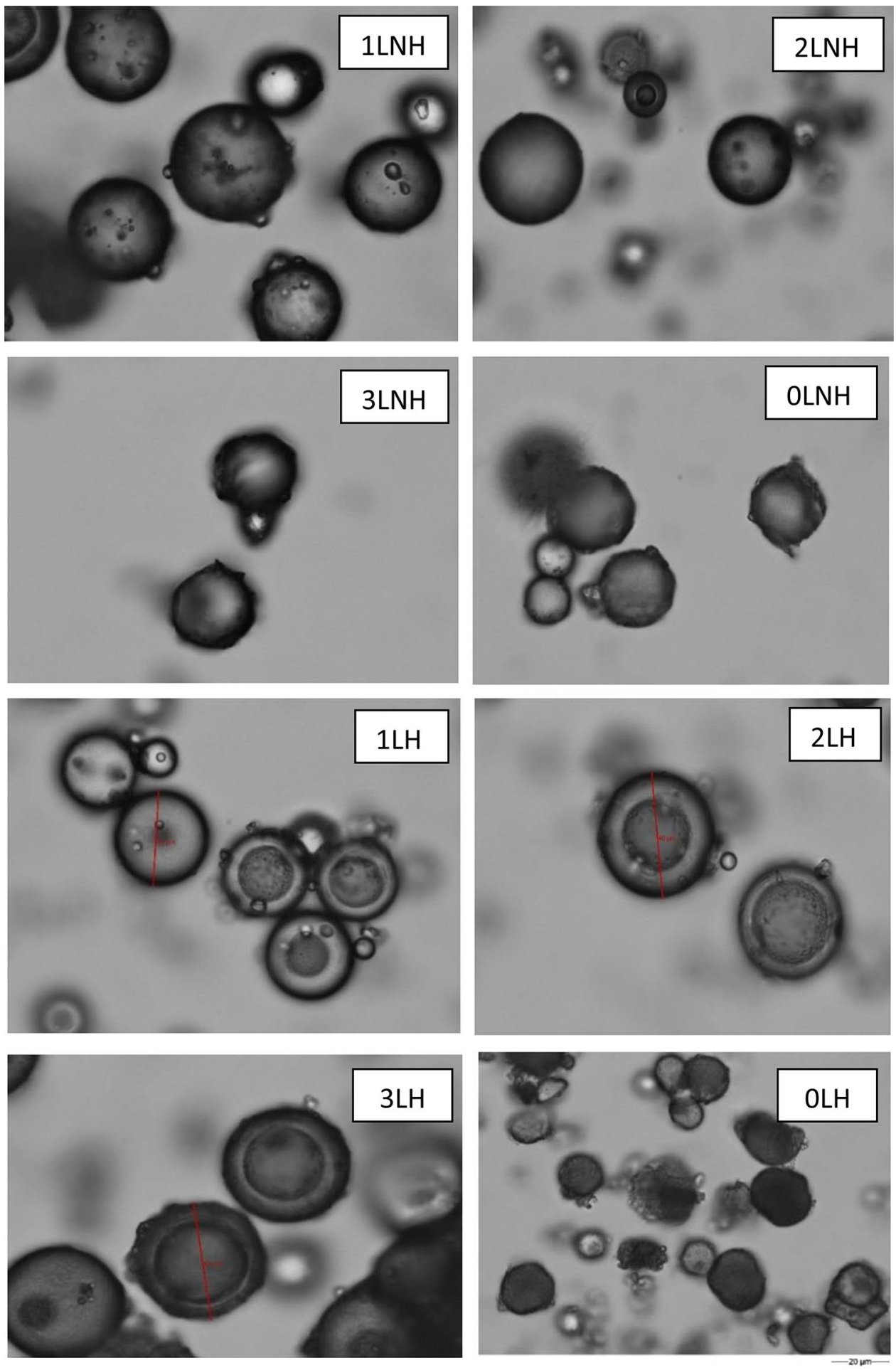

these material swell up to $1 \%$ and next does not swell more. Similar relationship is observed in acetone.

The results of the SPE experiments are presented in Fig. 7. Among the non-hybrid microspheres the highest values of recovery were obtained for the $1 \mathrm{LNH}$ copolymer (up to $90 \%$ for $2,4,6-\mathrm{TFPh}$ ). The homopolymer of $0 \mathrm{LNH}$ has a more developed specific surface area than the $1 \mathrm{LNH}$ copolymer. Despite this the recovery values were lower for OLNH than for $1 \mathrm{LNH}$. The addition of lignin to the sorbent improves its sorption capabilities towards phenol and its fluorinated derivatives. Sorption of phenolic compounds is a complex process influenced by many factors. When the functional groups are introduced to the polymeric material, its surface is more hydrophilic, the retention of the polar 
Table 3 Porous structure parameters of the studied copolymers

\begin{tabular}{lllll}
\hline Polymer & $\mathrm{S}_{\text {BET }}\left(\mathrm{m}^{2} / \mathrm{g}\right)$ & $\mathrm{V}_{\text {TOT }}\left(\mathrm{cm}^{3} / \mathrm{g}\right)$ & $\mathrm{D}_{\mathrm{A}}(\AA)$ & $\mathrm{D}_{\mathrm{MP}}(\AA)$ \\
\hline $1 \mathrm{LNH}$ & 305 & 0.672 & 88.2 & $4 / 32$ \\
$2 \mathrm{LNH}$ & 8 & 0.013 & 60.2 & $25 / 38$ \\
$3 \mathrm{LNH}$ & 2 & 0.005 & 117.2 & $35 / 260$ \\
0LNH & 542 & 1.299 & 95.6 & $35 / 320$ \\
$1 \mathrm{LH}$ & 522 & 1.881 & 138.1 & $33 / 360$ \\
$2 \mathrm{LH}$ & 471 & 1.274 & 103.9 & $34 / 250$ \\
$3 \mathrm{LH}$ & 454 & 1.125 & 95.2 & $35 / 240$ \\
0LH & 460 & 1.040 & 86.4 & $35 / 340$ \\
\hline
\end{tabular}

$S_{B E T}$ specific surface area, $V_{T O T}$ total pore volume, $D_{A}$ average pore diameter, $D_{M P}$ the most probable pore diameter

phenols is increased and that results in the improvement of sorption efficiency (Sobiesiak 2017). On the other hand, with the increasing lignin content the decrease in the recovery values is observed. The low effectiveness of $2 \mathrm{LNH}$ and $3 \mathrm{LNH}$ in phenols sorption is the result of their very low specific surface areas. For this reason, the optimization of the synthesis should be performed to obtain a more developed porous structure.

Lignin possesses a large number of oxygen-containing functional groups (methoxyl, hydroxyl, carbonyl). Comparing the recovery values for the $2 \mathrm{LNH}$ and $3 \mathrm{LNH}$ copolymers (up to $53 \%$ and $69 \%$, respectively) the increase is observed, although the specific surface areas are very small ( 8 and $2 \mathrm{~m}^{2} / \mathrm{g}$, respectively). In this case strong influence of the polar moieties from lignin becomes important factor determining the effectiveness of the sorption process.

Among the hybrid microspheres, the highest values of recovery (up to $90 \%$ for $2,4,6-\mathrm{TFPh}$ ) were obtained for the $1 \mathrm{LH}$ copolymer, and the lowest for the $0 \mathrm{LH}$ copolymer.

The highest recovery values were obtained for 2,4,6-TFPh and the lowest ones for $\mathrm{Ph}$ for both types of sorbents. If the electron withdrawing substituents are attached to the phenol molecule, it results in its stronger affinity to the polymeric material in adsorption processes.

The sorption abilities of a sorbent are characterized also by a breakthrough volume. The higher the breakthrough volume is, the better sorption ability the sorbent possesses
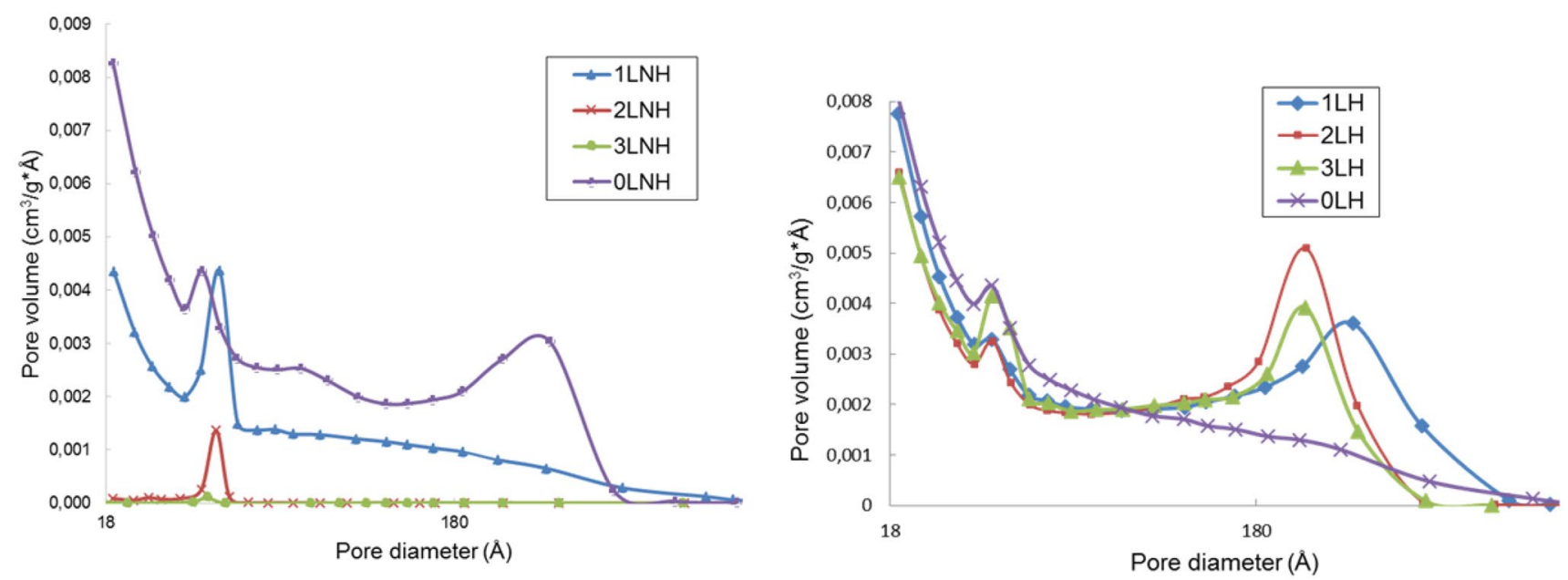

Fig. 5 Pore size distribution curves of the synthesized copolymers

Table 4 Swelling studies

\begin{tabular}{|c|c|c|c|c|c|c|c|}
\hline \multirow[t]{2}{*}{ Polymer } & \multicolumn{7}{|c|}{ Swellability coefficient, B (\%) } \\
\hline & Acetone & THF & Chloroform & $\mathrm{ACN}$ & Methanol & Toluene & Aqua dest. \\
\hline $1 \mathrm{LNH}$ & 17 & 5 & 12 & 11 & 17 & 24 & 0 \\
\hline $2 \mathrm{LNH}$ & 257 & 243 & 186 & 157 & 129 & 171 & 0 \\
\hline $3 \mathrm{LNH}$ & 225 & 175 & 200 & 183 & 157 & 163 & 0 \\
\hline OLNH & 27 & 27 & 27 & 17 & 0 & 9 & 0 \\
\hline $1 \mathrm{LH}$ & 4 & 7 & 6 & 5 & 7 & 13 & 8 \\
\hline $2 \mathrm{LH}$ & 7 & 13 & 13 & 8 & 6 & 6 & 6 \\
\hline $3 \mathrm{LH}$ & 7 & 13 & 15 & 5 & 6 & 8 & 6 \\
\hline OLH & 0 & 0 & 0 & 0 & 0 & 0 & 0 \\
\hline
\end{tabular}

$T H F$ tetrahydrofuran, $A C N$ acetronitrile 
AQUA DEST.

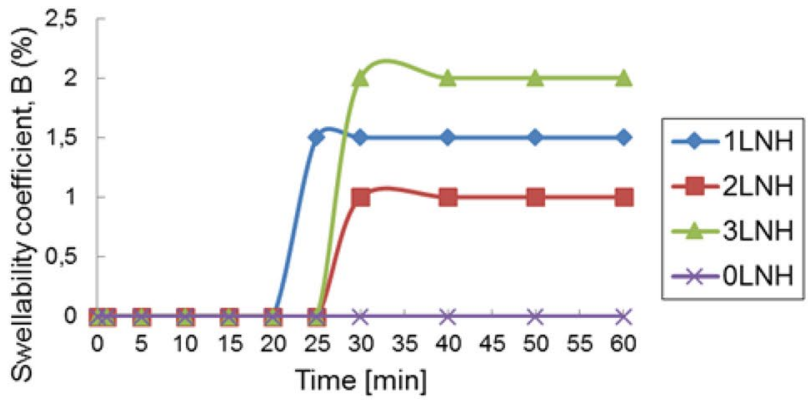

AQUA DEST.

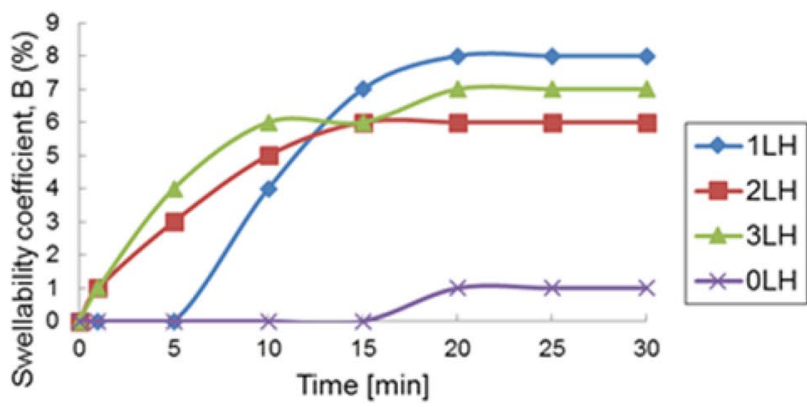

Fig. 6 Swelling studies

(Podkościelna et al. 2015). For the sorbents without TEVS with the increasing content of lignin in the sorbent, the breakthrough volume is shifted towards lower values. The addition of TEVS into the sorbents causes the increase in porous structure parameters, as a consequence sorption ability rise was observed. Therefore the breakthrough process proceeded more slowly and the breakthrough volume shifted towards higher values.

\section{Conclusions}

Proposed materials in form of polymeric microspheres possess in their structure biocomponent -lignin. To increase their porosity, triethoxyvinylsilane as a comonomer was applied. Microspheres porosity was investigated using the low-temperature nitrogen adsorption method. Synthesized materials are proved to be mesoporous. In comparison to non-hybrid materials, the increase of specific surface area in copolymers with TEVS is clearly visible. The well-developed specific surface area and the presence of the functional groups in the structure suggested that the studied materials should exhibit some sorption abilities. The obtained microspheres were tested as a sorbents using the SPE technique. The sorption of phenolic

\section{ACETONE}
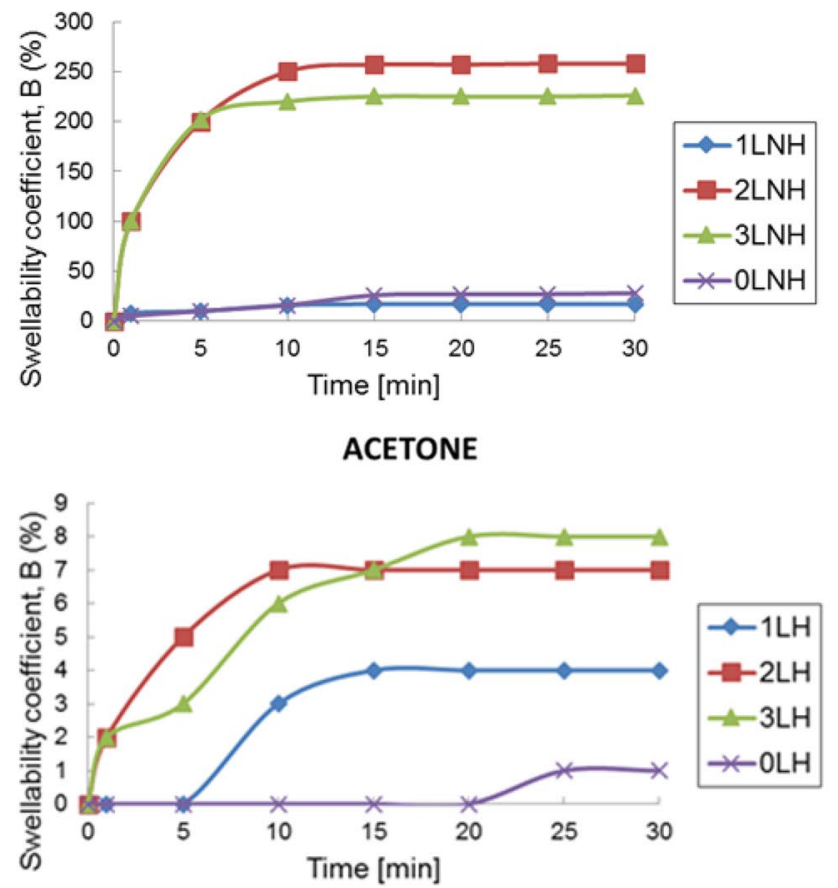

compounds (fluorophenols) on sorbents with lignin is affected by many factors. Although the big lignin molecule blocks the pores in the sorbents and the specific surface area decreases, the polar moieties from lignin promote sorption and increase its effectiveness. The largest recovery values were obtained for the materials with $17 \mathrm{w} / \mathrm{w} \%$ $(1 \mathrm{LNH})$ and $12 \mathrm{w} / \mathrm{w} \%(1 \mathrm{LH})$ of lignin for non-hybrid and hybrid systems, respectively. There was observed a correlation between the significance of developed porosity, and the amount of lignin as a source of functional groups. This amount is not enough to block the pores but enough to provide functional groups what promotes sorption processes. For the sorbents with TEVS the breakthrough volume is shifted towards higher values because of their developed specific surface areas which results in good sorption abilities. The SPE experiments proved quite high efficiency of the synthesized materials in removal of fluorophenols, (up to $90 \%$ for $2,4,6-\mathrm{TFPh}$ ) from the aqueous solutions as well as in the elution processes.

The swellability coefficient in common organic solvents and distilled water was determined. The significant reduction in swelling was observed for the copolymers with TEVS compared to those without this component. It can be the result of TEVS non-wettability in organic solvents and water. 
1LNH

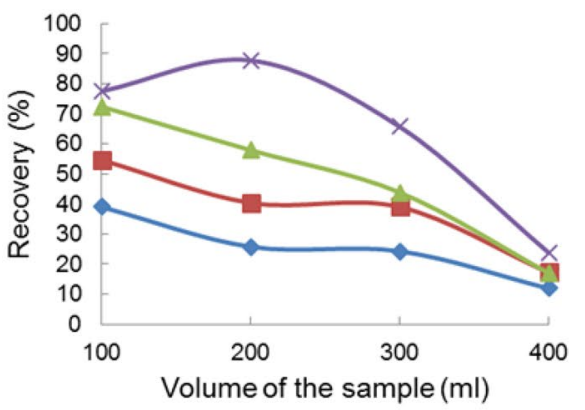

3LNH

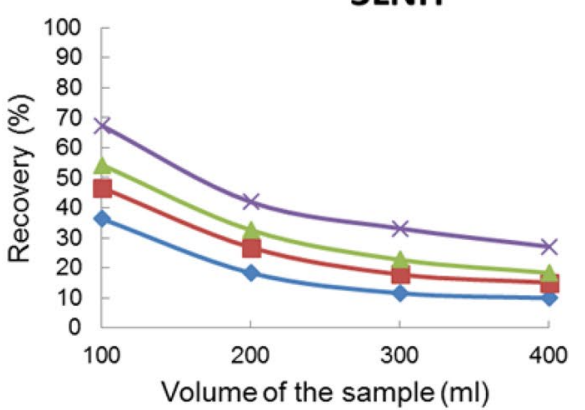

$1 \mathrm{LH}$

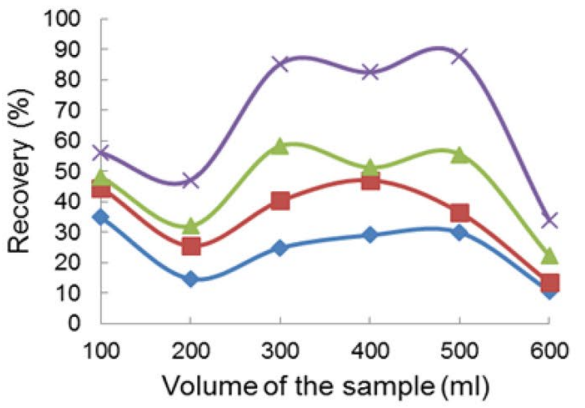

3LH

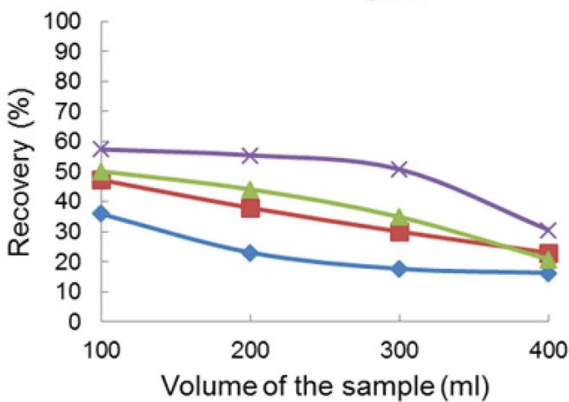

2LNH

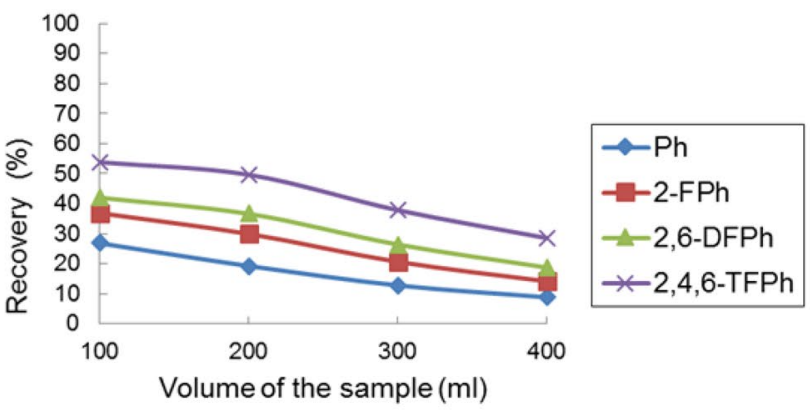

OLNH

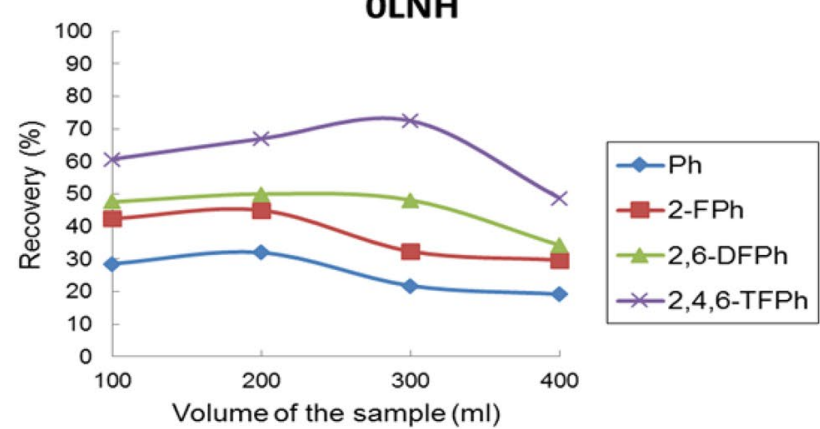

2LH

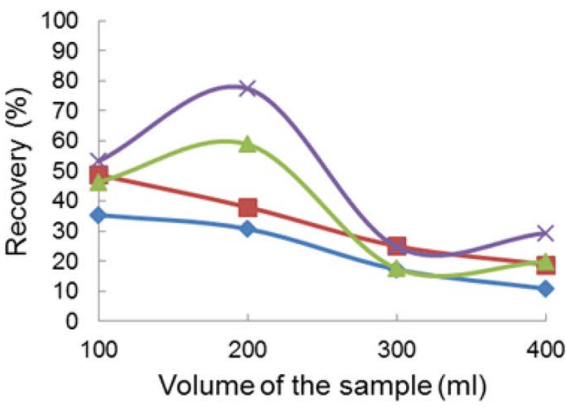

OLH

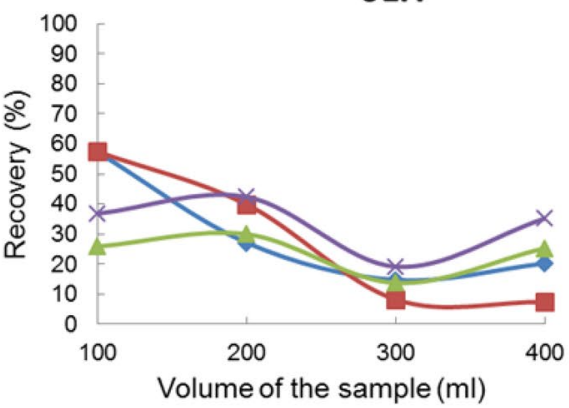

$\leadsto$ Ph

$-2-\mathrm{FPh}$

$\rightarrow-2,6-\mathrm{DFPh}$

$\rightarrow 2,4,6-T F P h$

Ph-phenol, FPh-2-fluorophenol, DFPh-2,6-difluorophenol, TFPh-2,4,6-trifluorophenol

Fig. 7 Results of solid phase extraction of phenols obtained for the presented materials 
Acknowledgements We would like to thank the COST Action CA17128 Establishment of a Pan-European Network on the Sustainable Valorization of Lignin for making it possible to exchange experience with the other scientists.

OpenAccess This article is distributed under the terms of the Creative Commons Attribution 4.0 International License (http://creativeco mmons.org/licenses/by/4.0/), which permits unrestricted use, distribution, and reproduction in any medium, provided you give appropriate credit to the original author(s) and the source, provide a link to the Creative Commons license, and indicate if changes were made.

\section{References}

Ahmaruzzaman, M., Sharma, D.K.: Adsorption of phenols from wastewater. J. Colloid Interface Sci. 287, 14-24 (2005)

Aksu, Z.: Application of biosorption for the removal of organic pollutants: a review. Process Biochem. 40, 997-1026 (2005)

Albadarin, A.B., Al-Muhtaseb, A.H., Al-laqtah, N.A., Walker, G.M., Allen, S.J., Ahmad, M.N.M.: Biosorption of toxic chromium from aqueous phase by lignin: mechanism, effect of other metal ions and salts. Chem. Eng. J. 169, 20-30 (2011)

Atifi, S., Miao, Ch, Hamad, W.Y.: Surface modification of lignin for applications in polypropylene blends. J. Appl. Polym. Sci. (2017). https://doi.org/10.1002/app.45103

Bartczak, P., Klapiszewski, Ł, Wysokowski, M., Majchrzak, I., Czernicka, W., Piasecki, A., Ehrlich, H., Jesionowski, T.: Treatment of model solutions and wastewater containing selected hazardous metal ions using a chitin/lignin hybrid material as an effective sorbent. J. Environ. Manag. 204, 300-310 (2017)

Braghiroli, F.L., Bouafif, H., Hamza, N., Neculita, C.M., Koubaa, A.: Production, characterization, and potential of activated biochar as adsorbent for phenolic compounds from leachates in a lumber industry site. Environ. Sci. Pollut. Res. 25, 26562-26575 (2018)

Carrott, P.J.M., Mourao, P.A.M., Ribeiro Carrott, M.M.L., Goncüalves, E.M.: Separating surface and solvent effects and the notion of critical adsorption energy in the adsorption of phenolic compounds by activated carbons. Langmuir 21, 1186311869 (2005)

Ciesielczyk, F., Bartczak, P., Klapiszewski, Ł, Jesionowski, T.: Treatment of model and galvanic waste solutions of copper(II) ionsusing a lignin/inorganic oxide hybrid as an effective sorbent. J. Hazard. Mater. 328, 150-159 (2017)

Cuia, J., Suna, H., Wangb, H., Suna, J., Niub, M., Wena, Z.: Preparation of siliceous lignin microparticles from wheat husks with a facile method. Ind. Crops Prod. 74, 689-696 (2015)

Da Silva, L.G., Ruggiero, R., Gontijo, P.D., Pinto, R.B., Royer, B., Lima, E.C., Fernandes, T.H., Calvete, T.: Adsorption of brilliant red $2 \mathrm{BE}$ dye from water solutions by a chemically modified sugarcane bagasse lignin. Chem. Eng. J. 168, 620-628 (2011)

Dąbrowski, A., Podkościelny, P., Hubicki, Z., Barczak, M.: Adsorption of phenolic compounds by activated carbon-a critical review. Chemosphere 58, 1049-1070 (2005)

Demirbas, A.: Adsorption of lead and cadmium ions in aqueous solutions onto modified lignin from alkali glycerol delignication. J. Hazard. Mater. B109, 221-226 (2004)

Demirbas, A.: Heavy metal adsorption onto agro-based waste materials: a review. J. Hazard. Mater. 157, 220-229 (2008)

Goliszek, M., Podkościelna, B., Fila, K., Riazanova, A.V., Aminzadeh, S., Sevastyanova, O., Gunko, V.M.: Synthesis and structure characterization of polymeric nanoporous microspheres with lignin. Cellulose 25, 5843-5862 (2018)
Klapiszewski, Ł, Królak, M., Jesionowski, T.: Silica synthesis by the sol-gel method and its use in the preparation of multifunctional biocomposites. Cent. Eur. J. Chem. 12(2), 173-184 (2014a)

Klapiszewski, Ł, Zdarta, J., Szatkowski, T., Wysokowski, M., Nowacka, M., Szwarc-Rzepka, K., Bartczak, P., Siwińska-Stefańska, K., Ehrlich, H., Jesionowski, T.: Silica/lignosulfonate hybrid materials: Preparation and characterization. Cent. Eur. J. Chem. 12(8), 719-735 (2014b)

Klapiszewski, Ł, Bartczak, P., Wysokowski, M., Jankowska, M., Kabat, K., Jesionowski, T.: Silica conjugated with kraft lignin and its use as a novel 'green' sorbent for hazardous metal ions removal. Chem. Eng. J. 260, 684-693 (2015)

Kumar, N.S., Asif, M., Al-Hazzaa, M.I.: Adsorptive removal of phenolic compounds from aqueous solutions using pine cone biomass: kinetics and equilibrium studies. Environ. Sci. Pollut. Res. 25, 21949-21960 (2018)

Mamleeva, N.A., Lunin, V.V.: Adsorption of Phenol on Wood Surfaces. Russ. J. Phys. Chem. A 90(3), 658-663 (2016)

Montaño, M., Gutleb, A.C., Murk, A.T.J.: Persistent toxic burdens of halogenated phenolic compounds in humans and wildlife. Environ. Sci. Technol. 47, 6071-6091 (2013)

Mourao, P.A.M., Carrott, P.J.M., Ribeiro Carrott, M.M.L.: Application of different equations to adsorption isotherms of phenolic compounds on activated carbons prepared from cork. Carbon $\mathbf{4 4}$, 2422-2429 (2006)

Navarro, A.E., Cuizano, N.A., Lazo, J.C., Sun-Kou, M.R., Llanos, B.P.: Comparative study of the removal of phenolic compounds by biological and non-biological adsorbents. J. Hazard. Mater. 164, 1439-1446 (2009)

Peterson, R.J.: Lignin: properties and applications in biotechnology and bioenergy (biotechnology in agriculture, industry and medicine). Nova Science Pub Inc, United Kingdom (2012)

Podkościelna, B., Sobiesiak, M.: Synthesis and characterization of organic-inorganic hybrid microspheres. Adsorption 22, 631-638 (2016)

Podkościelna, B., Sobiesiak, M., Zhao, Y., Gawdzik, B., Sevastyanova, O.: Preparation of lignin-containing porous microspheres through the copolymerization of lignin acrylate derivatives with styrene and divinylbenzene. Holzforschung 69(6), 769-776 (2015)

Podkościelna, B., Goliszek, M., Sevastyanova, O.: New approach in the application of lignin for the synthesis of hybrid materials. Pure Appl. Chem. 89, 161-171 (2017a)

Podkościelna, B., Gordobil, O., Riazanova, A.V., Dobele, G., Labidi, J., Lindstrom, M.E., Gun'ko,[V.M., Sevastyanova, O.: Novel porous materials obtained from techznical lignins and their methacrylate derivatives copolymerized with styrene and divinylbenzene. Chemistry Select 2, 2257-2264 (2017b)

Puziy, A.M., Poddubnaya, O.I., Gawdzik, B., Sobiesiak, M., Reinish, C.A., Tsyba, M.M., Segeda, T.P., Danylenko, M.I.: Nanostructured carbons for solid phase extraction. Appl. Surf. Sci. 256, 5216-5220 (2010)

Rajput, V.D., Yanning, Ch: Biosorption of heavy metals using agrowaste: a review. Pollut. Res. 34(1), 31-38 (2015)

Rodriguez, I., Llompart, M.P., Cela, R.: Solid-phase extraction of phenols-review. J. Chromatogr. A 885, 291-304 (2000)

Sciban, M.B., Klasnja, M.T., Antov, M.G.: Study of the biosorption of different heavy metal ions onto Kraft lignin. Ecol. Eng. 37, 2092-2095 (2011)

Severtson, S.J., Banerjee, S.: Sorption of Chlorophenols to Wood Pulp. Environ. Sci. Technol. 30, 1961-1969 (1996)

Sobiesiak, M.: Chemical structure of phenols and its consequence for sorption processes.” In: Soto-Hernandez, M., Palma-Tenango, M., Gracia-Mateos, M.R. (eds.) Phenolic Compounds-Natural Sources, Importance and Applications, pp. 3-28. InTech (2017)

Sobiesiak, M., Gawdzik, B., Puziy, A.M., Poddubnaya, O.I.: Polymer-based carbon adsorbents obtained from copolymer of 
4,4 -Bis(maleimidodiphenyl)methane and divinylbenzene for use in SPE. Chromatographia 64, 175-181 (2006)

Sostar-Turk, S., Simonic, M., Petrinic, I.: Wastewater treatment after reactive printing. Dyes Pigm. 64, 147-152 (2005)

Stoeckli, F., Lopez-Ramon, M.V., Moreno-Castilla, C.: Adsorption of phenolic compounds from aqueous solutions, by activated carbons, described by the Dubinin-Astakhov equation. Langmuir 17, 3301-3306 (2007)

Suhas, P.J., Carrott, M.R.: Lignin-from natural adsorbent to activated carbon: a review. Bioresour. Technol. 98, 2301-2312 (2007)

Torres, E., Bustos-Jaimes, I., Borgne, L.S.: Potential use of oxidative enzymes for the detoxification of organic pollutants. Appl. Catal. B 46, 1-15 (2003)

Veglio, F., Beolchini, F.: Removal of metals by biosorption: a review. Hydrometallurgy 44, 301-316 (1997)
Wu, J., Yu, H.Q.: Biosorption of phenol and chlorophenols from aqueous solutions by fungal mycelia. Process Biochem. 41, 44-49 (2006)

Wysokowski, M., Klapiszewski, Ł, Moszyński, D., Bartczak, P., Szatkowski, T., Majchrzak, I., Siwińska-Stefańska, K., Bazhenov, V.V., Jesionowski, T.: Modification of chitin with kraft lignin and development of new biosorbents for removal of cadmium(II) and nickel(II) ions. Mar. Drugs 12, 2245-2268 (2014)

Xu, G., Yan, G., Zhang, J.: Lignin as coupling agent in EPDM rubber: thermaland mechanical properties. Polym. Bull. 72, 2389-2398 (2015)

Yu, J.G., Zhao, X.H., Yang, H., Chen, X.H., Yang, Q., Yu, L.Y., Jiang, J.H., Chena, X.Q.: Aqueous adsorption and removal of organic contaminants by carbon nanotubes-review. Sci. Total Environ. 482-483, 241-251 (2014) 